\title{
Doença de Gaucher e atuação do cirurgião-dentista: relato de caso
}

\author{
Gaucher's disease and dentist surgery work: case report \\ Enfermedad de Gaucher y actuación del odontólogo: reporte de caso \\ Hugo Alex Paes da COSTA ${ }^{1}$ \\ Eliane de Oliveira Aranha RIBEIRO² \\ Gimol Benchimol de Resende PRESTES ${ }^{3}$ \\ Keuly Sousa SOARES ${ }^{4}$
}

\begin{abstract}
Graduando em Odontologia, Escola Superior de Ciências da Saúde,Universidade do Estado do Amazonas - UEA, 69065-001 Manaus-AM, Brasil ${ }^{2}$ Professora Mestre da Escola Superior de Ciências da Saúde, Universidade do Estado do Amazonas - UEA, 69065-001 Manaus-AM, Brasil ${ }^{3}$ Professora Doutora da Escola Superior de Ciências da Saúde, Universidade do Estado do Amazona - UEA, 69065-001 Manaus-AM, Brasil ${ }^{4}$ Professora Especialista da Escola Superior de Ciências da Saúde, Universidade do Estado do Amazonas - UEA, 69065-001 Manaus-AM, Brasil
\end{abstract}

\section{Resumo}

A Doença de Gaucher (DG) é uma doença de depósito lisossômico com origem genética de herança autossômica recessiva. São descritos três principais tipos de DG: Tipo I ou não neuropática, Tipo II ou neuropática aguda e Tipo III ou neuropática subaguda ou forma juvenil neuropática. O objetivo deste trabalho é descrever as características clínicas da DG e a realização do tratamento odontológico em um paciente hospitalizado com esta patologia. Paciente LFS, de 3 anos, gênero masculino internado no Pronto Socorro da Criança (PSC) Zona Sul, com diagnóstico de DG Tipo II. No exame físico foi observado hepatoesplenomegalia, traqueostomia, retardo no crescimento e atraso mental. No exame intraoral verificou-se higiene bucal deficiente com cáries nos elementos 51, 52, 61, 62, 74, 75, 84 e 85. Como o paciente encontrava-se internado e os focos infecciosos odontológicos poderiam estar influenciando na saúde geral do paciente os procedimentos foram realizados sob anestesia geral no centro cirúrgico. Os elementos 52, 51, 61, 62 e 84 foram extraídos e os outros elementos foram restaurados pela técnica do ART. Cabe ao cirurgião-dentista integrar-se a equipe multidisciplinar hospitalar envolvida no tratamento destes, conhecer a patologia e proporcionar além de saúde bucal a melhoria da saúde geral do paciente hospitalizado com DG.

Descritores: Doença de Gaucher; Pessoas com Deficiência; Unidade Hospitalar de Odontologia.

\begin{abstract}
Gaucher's Disease (GD) is a lysosomal reservoir disease with a genetic origin of autosomal recessive in heritance. Three major types of GD are described: Type I or non-neuropathic, Type II or acute neuropathic and Type III neuropathic subacute or juvenile neuropathic form. The aim of this study was to describe the clinical characteristics of the GD and dental treatment management in a patient hospitalized with this pathology. Patient LFS, 3 years old, male gender, hospitalized in the South Zone Pediatric Emergency, with diagnosis GD Type II. At physical examination were observed hepatosplenomegaly, tracheostomy, growth retardation and mental retardation. In the oral examination, poor oral hygiene was found and decay at $51,52,61,62,74,75,84$ e 85 . As patient was hospitalized and the dental infectious focus could be influencing the general health of the patient, the procedures were performed under general anesthesia in the surgical center. Elements 52, 51, 61, 62 and 84 were extracted and the other elements were filling by the ART technique. It is up to the dental surgeon to integrate the multidisciplinary hospital team involved in the treatment of these, to know the pathology and to provide besides oral health the improvement of the general health of the patient hospitalized with GD.
\end{abstract}

Descriptors: Gaucher Disease; Disabled Persons; Dental Service, Hospital.

\section{Resumen}

La enfermedad de Gaucher (DG) es una enfermedad de depósito lisosómico con origen genético de herencia autosómica recesiva. Se describen tres principales tipos de DG: Tipo I o no neuropática, Tipo II o neuropática aguda y Tipo III neuropática subaguda o forma juvenil neuropática. El objetivo de este trabajo describir las características clínicas de la DG y la realización del tratamiento odontológico en un paciente hospitalizado con esta patología. Paciente LFS, de 3 años, género masculino internado en el Pronto Socorro del Niño Zona Sur, con diagnóstico de DG Tipo II. En el examen físico se observó hepatoesplenomegalia, traqueostomía, retraso en el crecimiento y retraso mental. En el examen intra oral se verificó higiene bucal deficiente con caries en los elementos 51, 52, 61, 62, 74, 75, 84 y 85 . Como el paciente se encontraba internado y los focos infecciosos odontológicos podrían estar influenciando en la salud general del paciente los procedimientos fueron realizados bajo anestesia general en el centro quirúrgico. Los elementos 52, 51, 61, 62 y 84 fueron extraídos y los otros elementos fueron restaurados por la técnica del ART. Se debe al cirujano-dentista integrarse al equipo multidisciplinario hospitalario involucrado en el tratamiento de éstos, conocer la patología y proporcionar además de salud bucal la mejoría de la salud general del paciente hospitalizado con DG.

Descriptores: Enfermedad de Gaucher; Personas con Discapacidad; Servicio Odontológico Hospitalario.

\section{INTRODUÇÃO}

No final do século XIX, na França o médico Philippe Gaucher descreveu de forma inédita, uma doença cujo paciente apresentava um aumento significativo do fígado e do baço após biópsia. Patologia essa que veio a se chamar Doença de Gaucher (DG) é uma doença de depósito lisossômico com origem genética de herança autossômica recessiva, que consiste no aumento de glicocerebrosídeo não digerido no interior dos macrófagos teciduais, o que leva a alterações histológicas principalmente em órgãos com grande quantidade de células do sistema imunológico como o fígado, baço e ossos ${ }^{1-5}$.

A DG faz parte das enfermidades raras ou pouco frequentes, mas dentre todas as doenças de depósito lisossômico é a mais comum e atinge todas as raças, com uma incidência de 1:50.000 nascidos vivos. Em judeus Ashkenazi essa incidência é maior, atingindo cerca de 1:400 nascidos vivos. O Brasil ocupa o terceiro lugar dentre os países com maior número de diagnósticos confirmados, atrás apenas dos Estados Unidos e Israel $^{3-7}$.

É uma doença causada por erros inatos do metabolismo (EIM) que são definidos como doenças causadas por bloqueio em uma ou mais vias metabólicas, afetando o seu funcionamento normal. A DG tem origem na mutação de dois alelos do gene GBA1 localizados no braço longo do cromossomo 
1 (região q21). O EIM se dá na mutação do gene que codifica a enzima Beta-glicocerebrosidase que é responsável pela hidrólise do glicocerebrosídeo em glicose e ceramida, a mutação leva a produção de uma enzima ineficiente e uma vez que a enzima se encontra mutada ou alterada não ocorre à síntese da molécula, causando a doença ${ }^{7-12}$.

São descritos três principais tipos de DG: tipos I, II, III. O Tipo I ou não neuronopática é a forma mais comum da doença, com freqüência de $90 \%$ a $95 \%$ dos casos. Neste tipo os depósitos de glicocerebrosídeo se restringem aos macrófagos do corpo sem envolvimento cerebral, o baço e ossos são evidenciados nesse tipo. Apesar de não haver envolvimento cerebral, os pacientes podem desenvolver sintomas neurológicos de forma secundária, além disso, podemos observar discreta atividade da enzima glicocerebrosidase $e^{6,7,10,11}$. As manifestações clínicas comuns da DG compreendem os seguintes sintomas: hepatoesplenomegalia, anemia, trombocitopenia, osteopenia, fraturas patológicas, dor óssea, retardo do crescimento. Pacientes com a DG do tipo I podem apresentar sintomas neurológicos secundários devido à compressão da medula espinhal ou raiz nervosa bem como infarto do baço e dor abdominal ${ }^{4,6,13}$. A DG Tipo II ou neuropática aguda é o padrão cerebral infantil agudo. Nesse tipo não é possível detectar atividade da glicocerebrosidase nos tecidos, o comprometimento progressivo do sistema nervoso central domina o aspecto clínico. A enfermidade se manifesta de forma aguda nos primeiros 4 a 5 meses de vida, é de rápida evolução, exibe clássica neurodegeneração, acompanhada de depósitos de lipídios nos neurônios insignificantes. Esse rápido e progressivo curso limita o desenvolvimento $\mathrm{e}$ acarreta a morte precoce ${ }^{6-8,10,12}$. As manifestações clínicas neuropáticas são predominantes, quadro neurológico grave com envolvimento bulbar (estridor, estrabismo e dificuldades para engolir), piramidal, comprometimento do baço, fígado e pulmões além de múltiplas convulsões, apneia e progressivo retardo mental ${ }^{3,6}$. O tipo III, forma neuropática subaguda ou forma juvenil neuropática, é intermediária entre os tipos I e II e tem início na infância, com sintomas mais característicos que o Tipo I somada ao envolvimento neurológico do Tipo II. O comprometimento neurológico do sistema nervoso central é de evolução lenta e progressiva e geralmente se inicia entre a adolescência e fase adulta, com expectativa de vida que varia entre a segunda e quarta década de vida ${ }^{7,19,12}$. No tipo III o comprometimento do baço, fígado e ossos são comparáveis ao tipo I, porém mais graves. O envolvimento neurológico acontece em idade avançada, normalmente com epilepsia, ataxia e disfunção neurológica progressiva, porém inferior ao tipo $\mathrm{II}^{6,7,9.13,14}$

O diagnóstico pode ser feito imediatamente após suspeitas clínicas por meio de exames laboratoriais ou genéticos: achados morfológicos, diagnóstico molecular ou dosagem enzimática. O diagnóstico morfológico é feito por meio da presença de células de Gaucher nas amostras analisadas que geralmente são obtidas na medula óssea. O diagnóstico molecular realizado por técnica de recombinação em cadeia de polimerase dos fragmentos genômicos e detecção das mutações específicas, definindo o genótipo ${ }^{1,21}$. O enzimático é apontado como o mais efetivo, baseando-se na medida da atividade enzimática da betaglicocerebrosidase, por ensaios fluorimétricos dos leucócitos ou outras células do sangue periférico, com valores de referência variáveis dependendo do laboratório ${ }^{1,6,7,15,16}$.

O tratamento adotado para a DG é a terapia de reposição enzimática (TRE) que surgiu na década de 80 , com a enzima beta-glicocerebrosidase associada a uma porção de carboidrato, a Alglucerase (Ceredase $($ )). Este foi o primeiro medicamento disponível no mercado em 1991, mas em 1994 foi substituída pela Imiglucerase (Cerezyme $\left.{ }^{\circledR}\right)$ enzima beta-glicocerebrosidase obtida por recombinação genética e marcada para o macrófago. Apesar de não haver consenso sobre a quantidade da enzima necessária para manter a reversão dos sintomas, ela se mostra eficaz na diminuição dos sintomas no fígado, baço, dor óssea, fraturas patológicas e no aumento da hemoglobina e plaquetas. O tratamento é indicado para o tipo I, sendo ineficaz nas manifestações neurológicas nos Tipos II e III, uma vez que a enzima não ultrapassa a barreira hematoencefálica ${ }^{2,6,8,16,17}$.

Como alternativa a terapia de reposição enzimática, existe a terapia de redução de substratos (TRS). Nessa terapia é usada a sustância Miglustat (Zavesca ${ }^{\circledR}$ ) que age inibindo a enzima glicosilceramida sintase, diminuindo a concentração do substrato ao nível que a enzima mutante consiga evitar o armazenamento patológico. As vantagens dessa substância são a via de administração oral e sua capacidade de atravessar a barreira hematoencefálica, graças ao baixo tamanho molecular, o que possibilita o tratamento da DG tipos I, II e $\mathrm{III}^{6,8,17,18}$.

$\mathrm{Na}$ área odontológica existem relatos de manifestações bucais como pigmentação amarela e petéquias na mucosa, assim como doença periodontal e gengival, mas sem comprovação da associação ${ }^{19-21}$. O envolvimento mandibular é geralmente assintomático com achados em exames de radiográficos de rotina. Pacientes jovens com DG podem apresentar erupção dentária tardia. Como sangramentos excessivos após exodontias são comuns, recomenda-se profilaxia contra diátese 
hemorrágica, mesmo em pacientes que fazem terapia de reposição enzimática ${ }^{19-24}$.

A presença do cirurgião-dentista no ambiente hospitalar se faz cada vez mais necessária, pois os pacientes hospitalizados muitas vezes são impossibilitados de manter uma higiene bucal adequada. $\mathrm{O}$ acompanhamento do $\mathrm{CD}$ torna-se indispensável na avaliação da presença do biofilme, cárie dentária, doença periodontal, lesões traumáticas entre outras, que apresentam risco ou desconfortos aos pacientes hospitalizados. Portanto, a presença do $C D$ na equipe multidisciplinar é de grande importância na terapêutica e qualidade de vida dos pacientes que se encontram nessa condição ${ }^{25-27}$.

Assim o objetivo deste relato de caso foi descrever as características clínicas da doença de Gaucher e a realização do tratamento odontológico em um paciente hospitalizado com a doença.

\section{CASO CLÍNICO}

Paciente com 3 anos de idade, gênero masculino, internado no PSC Zona Sul, com diagnóstico de DG Tipo II, apresentando pneumonia, bronco displasia e úlcera subglótica (Figura 1). Esteve internado na UTI por 46 dias, depois transferido para semi-intensiva. Devido à má condição de saúde bucal foi solicitada a presença do cirurgião-dentista. $\mathrm{Na}$ anamnese foi observado que o paciente faz uso de Imiglucerase (Cerezyme $\left.{ }^{\circledR}\right)$, para suprimir as manifestações da doença de Gaucher, Aerolin ${ }^{\circledR} 100 \mathrm{mg}$, Clenil® 250mg.

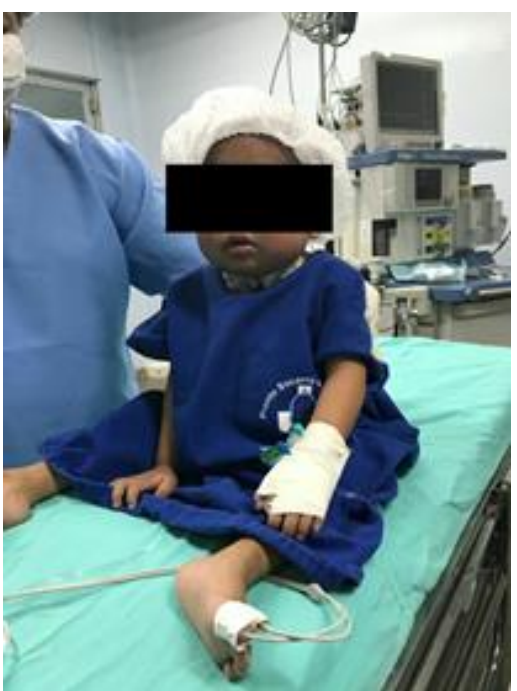

Figura 1: Paciente com diagnóstico de Doença de Gaucher tipo II.

No exame clínico foi observada hepatoesplenomegalia, traqueostomia, retardo no crescimento e atraso mental (Figura 2). No exame intra oral observamos higiene bucal deficiente com cáries nos elementos 52, 51, 61, 62, 75, 74, 84 e 85 . (Figuras 3, 4 e 5) Como o paciente se encontrava internado $e$ os focos infecciosos odontológicos poderiam influenciar na saúde geral do paciente, os procedimentos foram realizados sob anestesia geral no centro cirúrgico. Os elementos 52, 51, 61, 62 e 84 foram extraídos e os outros elementos foram restaurados pela técnica do ART (Figuras 7 e 8) restabelecendo a saúde bucal do paciente.

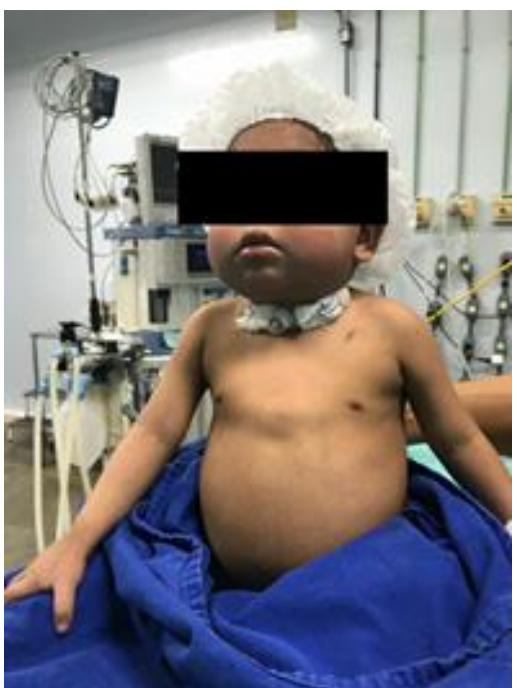

Figura 2: Paciente com hepatoesplenomegalia e traqueostomia.

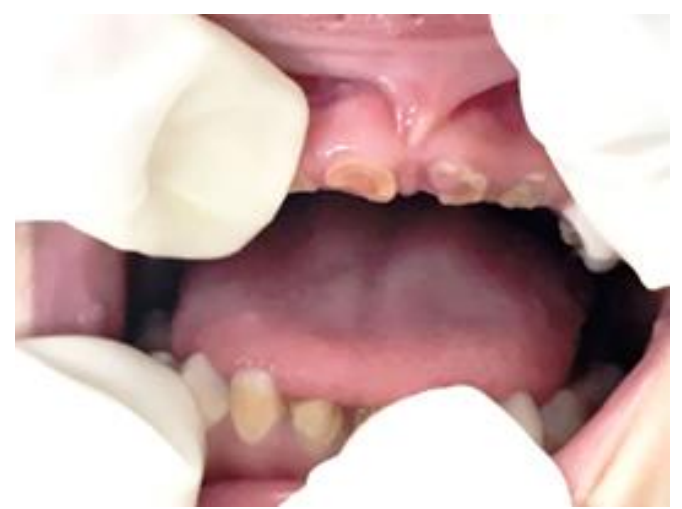

Figura 3: Elementos cariados 51, 52, 61 e 62

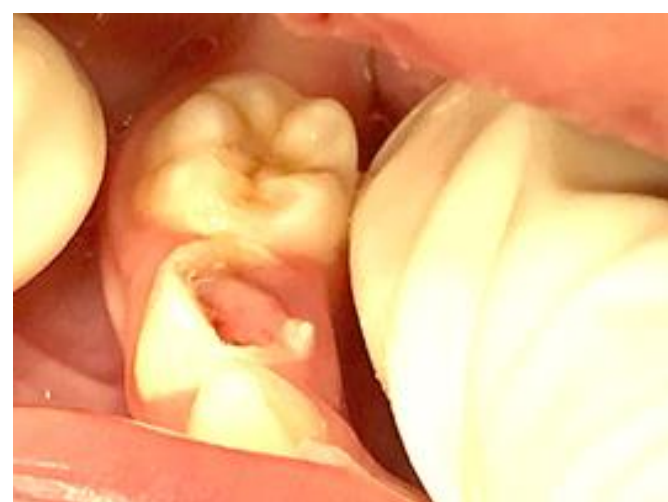

Figura 4: Elementos cariados 84 e 85.

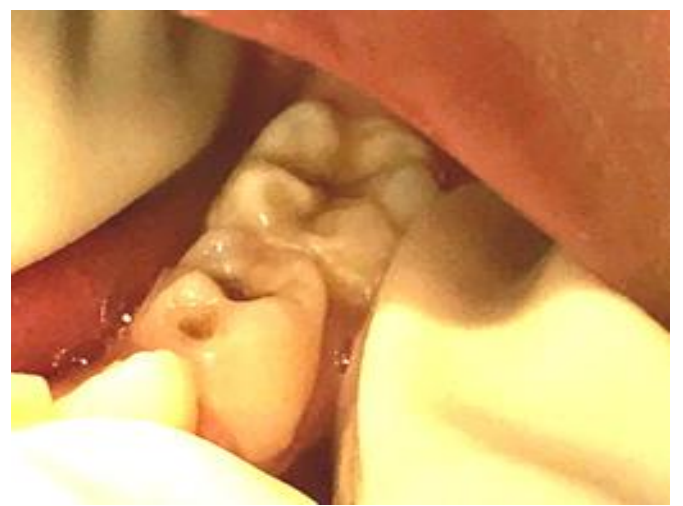

Figura 5: Elementos cariados 74 e 75. 


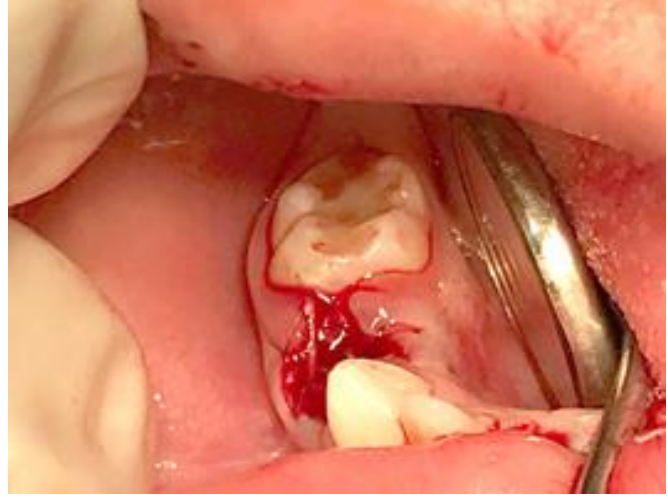

Figura 7: Exodontia do elemento 84 e restauração do 85 .

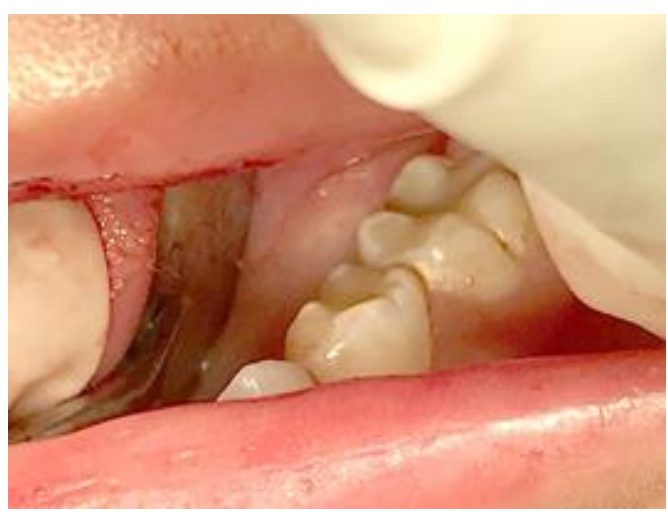

Figura 8: Restauração dos elementos 74 e 75.

\section{DISCUSSÃO}

No paciente com DG tipo II observa-se o envolvimento pulmonar que é uma característica marcante, pois leva à morte nos primeiros dois anos de vida, o que vem de encontro, em parte, com o relato apresentado, pois o paciente fez uso de respirador mecânico e medicação para as doenças do trato respiratório, mas a idade do nosso paciente supera a media da expectativa de vida, o que leva ao questionamento se foi a TRE e/ou as medidas paliativas que prolongaram sua expectativa de vida ${ }^{9}$.

Neste relato, o paciente apresenta retardo do crescimento e atraso mental, corroborando com trabalhos que citam essa característica como sendo padrão para DG tipo $\mathrm{II}^{1,6}$.

O presente trabalho aponta a importância do $\mathrm{CD}$ em ambiente hospitalar, devido à incapacidade de uma higiene bucal adequada que contribui para o crescimento de bactérias e fungos na cavidade bucal, favorecendo o surgimento de infecções, que influenciam no bem estar do paciente. A presença do CD se mostra uma alternativa acessível, de fácil execução, necessária e de extrema relevância.

Neste relato, a presença do CD foi solicitada, o que permitiu a identificação de focos de infecção bem como a realização do tratamento odontológico em nível hospitalar, favorecendo a melhora das condições de saúde geral do paciente ${ }^{25-27}$.

A TRE não combate com eficácia a DG do tipo II, pois a enzima não atravessa a barreira hematoencefálica. Sua eficácia se restringe apenas ao tratamento do tipo I, tipo não neuropático ${ }^{8,24}$. Um contraponto ao caso relatado, pois o paciente faz uso da TRE.

\section{CONSIDERAÇÕES FINAIS}

A doença de Gaucher é uma doença rara, com origem genética, de herança autossômica recessiva. $\mathrm{O}$ cirurgião-dentista deve ficar atento às características clinicas da DG e suas possíveis interferências para tratamento odontológico. Apesar do envolvimento dentário não ser comum, o comprometimento mandibular, maxilar $\mathrm{e}$ as alterações hematológicas são significantes aos procedimentos odontológicos e merecem atenção. Portanto cabe ao cirurgião-dentista integrar-se a equipe multidisciplinar hospitalar envolvida no tratamento de pacientes acometidos com a doença, proporcionando, além de promoção da saúde bucal, melhora da saúde geral do paciente hospitalizado com DG.

\section{REFERÊCIAS}

1. Silva TU, da Cunha MAA, Lins JP. Doença De Gaucher: levantamento epidemiológico no Distrito Federal. XIII International Conference on Engineering and Technology Eduacation. 1619 march 2014; Guimarães, Portugal.

2. Oi SSP, Nicolau DI, Santos SKA, Silva MACN, Viana GMC. Gaucher disease in a family from Maranhão. Rev Bras Hematol Hemoter. 2014; 36(5):373-78.

3. Freitas SEO, Ferreira TTC, Costa BGS, Soares RM, Lucena NC, Correia NB. Qualidade de vida de pacientes com doença de Gaucher. Rev Enferm UFPE 2017; 11(11):4282-88.

4. Coelho JC, Garcia CS, Santin J. Doença de Gaucher: um ano de triagem e diagnóstico. $34^{\mathrm{a}}$ Semana Científica do Hospital de Clínicas de Porto Alegre. 1-5 set 2014; Porto Alegre, Brasil.

5. Rizzon LR, Basgalupp SP, Siebert M, Vairo FP, Schwartz IVD. Análise de mutações no gene GBA1 em pacientes com Doença de Gaucher. Feira de inovação tecnológica da UFRGS FINOVA. 12-16 set 2016; Porto Alegre, Brasil.

6. Vairo FP. O sistema imune na doença de Gaucher [tese]. Porto Alegre: Universidade Federal do Rio Grande do Sul; 2016.

7. Ministério da Saúde: Secretaria de Atenção à Saúde (Brasil). Portaria No 1.266, de 14 de novembro de 2014. Aprova o protocolo clínico e diretrizes terapêuticas da doença de Gaucher. Diário Oficial da União 18 nov 2014; Seção 1.

8. Tirelli KM. Estudo das características bioquímicas da $\beta$-glicosidase humana em indivíduos homozigotos e heterozigotos para a doença de Gaucher com mutações prédeterminadas: comparação com indivíduos normais [tese]. Porto Alegre: Universidade Federal do Rio Grande do Sul; 2005. 
9. Martins AM, Lobo CL, Sobreira EAP, Valadares ER, Porta G, Semionato Filho J et al. Tratamento da doença de Gaucher: um consenso brasileiro. Rev Bras Hematol Hemoter. 2003; 25(2):89-95.

10. Kumar V, Abbas AK, Fausto N (eds). Robbins e Cotran - Patologia: Bases patológicas das Doenças. 8. ed. Rio de Janeiro: Elsevier; 2010. p. 153.

11. Mello RAF, Mello MBN, Pessanha LB. Ressonância magnética e o escore $\mathrm{BMB}$ na avaliação do acometimento ósseo em pacientes com doença de Gaucher. Radiol Bras. 2015; 48(4):216-19.

12. Pastores GM, Hughes DA. Gaucher Disease. Gene Reviews®. 2000. In: Adam MP, Ardinger HH, Pagon RA, et al. (eds). GeneReviews ${ }^{\circledR}$. Seattle (WA): University of Washington, Seattle; 1993-2018. Available from: https:// www.ncbi. nlm. nih.gov/books/NBK1269/.

13. Pessanha LB. Ressonância magnética na avaliação do acometimento ósseo na doença de Gaucher tipo I [dissertação]. Vitória: Universidade Federal do Espírito Santo; 2016.

14. Bertholdo DB. Correlação do envolvimento cerebral através de espectroscopia de prótons por ressonância magnética com gravidade de doença óssea em pacientes com doença de Gaucher Tipo I [dissertação]. Curitiba: Universidade Federal do Paraná; 2016.

15. Camelo Júnior JS, Dragosky M, Drelichman G. Doença de Gaucher Tipo I no esqueleto: revisão da América Latina. Coluna/Columna. 2016; 15(4):317-24.

16. Sobreira EAP, Bruniera P. Avaliação de dois anos de tratamento da doença de Gaucher tipo I com terapia de reposição enzimática em pacientes do estado de São Paulo, Brasil. Rev Bras Hematol Hemoter. 2008; 30(3):193-201.

17. Cerezyme [bula]. Allston; Genzyme Corporation

18. Zavesca [bula].Grenzach-Wyhlen; Actelion Manufacturing $\mathrm{GmbH}$.

19. Gambareli FR, Avaliação das condições bucais, da qualidade de vida e das características craniofaciais em indivíduos com doença de Gaucher [tese]. Piracicaba: Faculdade de Odontologia de Piracicaba, Universidade Estadual de Campinas; 2009

20. Horwitz J, Hirsh I, Machtei EE. Oral aspects of Gaucher's disease: a literature review and case report. J Periodontol. 2007; 78(4):783-88.

21. Fischman SL, Elstein D, Sgan-Cohen H, Mann J, Zimran A. Dental profile of ients with Gaucher disease. BMC Oral Health 2003; 3:4.

22. Wasserstein MP, Martignetti JA, Zeitlin R, Lumerman H, Solomon M, Grace ME et al. Type 1 Gaucher disease presenting with extensive mandibular lytic lesions: Identification and expression of a novel acid $\beta$-glucosidase mutation. Am J Med Genet. 1999; 84(4):334-39.

23. Saranjam HR, Sidransky E, Levine WZ, Zimran A, Elstein D. Mandibular and dental manifestations of Gaucher disease. Oral Dis. 2012; 18(5):421-29.

24. Lisboa GM, Guedes VL. Exodontia in patient with Gaucher's disease. Rev Bras Hematol Hemoter. 2011; 33(6):481-82.

25. Gomes SF, Esteves MCL. Atuação do cirurgiãodentista na UTI: um novo paradigma. Rev bras odontol. 2012; 69(1):67-70

26. Andrade APP, Eleutéio ASL. Pacientes portadores de necessidades especiais: abordagem odontológica e anestesia geral. Rev bras odontol. 2015; 72(1/2):66-9.

27. Assis C. Atendimento odontológico nas UTIs. Rev bras odontol. 2012. 69(1):72-5

\section{CONFLITO DE INTERESSES}

Os autores declaram não haver conflitos de interesse.

\section{AUTOR PARA CORRESPONDENCIA}

\section{Gimol Benchimol de Resende Prestes}

gresende@uea.edu.br

Submetido em 18/04/2018

Aceito em 06/06/2018 